\title{
Pastorale Überlegungen zur Kommunikation
}

\section{von Paul Nikolajczyk}

Während sich ein älteres deutschsprachiges Großlexikon mit ganzen eineinhalb Zeilen beim Stichwort "Kommunikation"' begnügt, ist vor allem seit dem Zweiten Weltkrieg die "Kommunikation" zu einem Forschungsobjekt geworden, das in nahezu alle Wissenschaftsbereiche eindringt. In einem bedeutenden Werk über affirmative Didaktik kommen die Autoren sogar zu der Feststellung “. . . man kann sagen, daß der Grad der Fortschrittlichkeit einer Gesellschaft an der Menge der Kommunikationen abgelesen werden kann" 2 . Um Kommunikation vollziehen zu können, nennt das zweite Vatikanische Konzil in seinem am 4. Dezember $1963^{\circ}$ verkündeten Dekret "Inter Mirifica" sowohl die Kenntnis der Grundsätze sittlicher Wertordnung als auch die Bereitschaft, sie hier zu verwirklichen ${ }^{3}$. Zwar bezieht sich dieser Text in erster Linie auf die Benutzung der "sozialen Kommunikationsmittel" wie Presse, Film, Rundfunk und Fernsehen, dennoch ist es gewiß legitim, die vom Konzil genannten Voraussetzungen auf andere und sogar auf alle Formen der Kommunikation zu übertragen. So sei daran erinnert, daß das Hirtenschreiben der Deutschen Bischöfe vom 22. September 1977 "Grundwerte verlangen Grundhaltungen" u.a. auch an die Fundamente jeglicher Kommunikation erinnert ${ }^{4}$.

Dieses ist umso notwendiger, weil vieles von dem, was noch vor wenigen Jahren als Selbstverständlichkeit galt (z.B. Glauben, Hoffen, Lieben, Klugheit, Gerechtigkeit, Tapferkeit, Zucht, sowie der Umgang mit den Strebungen nach Besitz, Ansehen, Genießen) inzwischen keineswegs mehr selbstverständlich ist. Damit ist nicht gesagt, daß frühere Generationen die genannten Selbstverständlichkeiten "beherrschten", wohl aber, daß sich inzwischen bei nicht wenigen Menschen eine Bewußtseinsveränderung vollzogen hat, so daß ein weitreichender Dissens über Grundwerte und Grundhaltungen das genannte Schreiben der Bischöfe notwendig machte. Damit diese Diskussion über Grundwerte und Grundhaltungen weitergeht, sei der folgende Beitrag als ein Diskussionsbeitrag verstanden. Mit Hilfe des noch zu erläuternden Heinen-Modells ${ }^{5}$ soll versucht werden, die Grundlagen der Kommunikation zu bestimmen und pastorale Hilfen zur personengemäßen Entfaltung der Kommunikationsfähigkeit für das Gelingen des lebenslangen menschlichen Werde- und Reifungsprozesses zu ermöglichen.

Wilhelm Heinen, der am 26. Februar 197970 Jahre alt wurde, war lange Zeit Professor und Seminardirektor für katholische Moraltheologie der Westfälischen WilhelmsUniversität in Münster. Er widmete sich besonders der Erforschung der Familie, weil Grundwerte und Grundhaltungen eingeübt, entfaltet und weitergegeben werden in einer "gesunden Ursprungsfamilie" (= Erzähl-gemeinschaft). Durch jahrelange Beobachtungen erkannte Heinen, wie wichtig für jede Kommunikation die personengemäße Begegnung ist. In seinem bedeutenden Werk "Werden und Reifen des Menschen in Ehe und Familie" 6 zeigt er auf, daß jeder Mensch während seines lebenslangen Werdeund Reifungsprozesses, der ja mit dem Erleben der Ursprungsfamilie beginnt, angewiesen ist auf "Beziehungs-Personen", die in folgende "acht Grundgestalten" eingeteilt werden: Vater, Mutter, Bruder, Schwester, Ehemann, Ehefrau, Sohn, Tochter oder deren Stellvertreter.

Paul Nikolajczyk, Diplomtheologe, studierte nach einer fünfjährigen Tätigkeit in der Industrie Theologie in Paderborn und Münster; Priesterweihe 1975: jetzt Pastor in Castrop-Rauxel. 
Heinen-Modell bedeutet daher: das personengemäße Erleben der "acht Grundgestalten" ist Voraussetzung für das Gelingen des lebenslangen menschlichen Werde- und Reifungsprozesses, wobei beim ungenügenden Erleben dieser "acht Grundgestalten" Reifungsrückstände auftreten. Ein solcher Mensch "fragt" nach den Möglichkeiten des Gelingens seines Lebens; diese Frage tritt auf in Form der "direkten Frage" (z.B. Gespräche) oder in Form der "indirekten Frage" (z.B. Flucht in verschiedene Krankheitsformen); Ziel dieser Fragen sind eine Aussöhnung mit den Grundgestalten, welche enttäuschten, weil nur so die zum Leben notwendige Kommunikation gelingen kann.

Dieses Heinen-modell soll hier nun entfaltet, verdeutlicht und als brauchbare pastorale Hilfe zur Kommunikation ausgewiesen werden.

\section{Die Bedeutung der Ursprungsfamilie}

Es darf davon ausgegangen werden, daß jeder Mensch normalerweise in eine Familie hineingeboren wird. In einzigartiger Weise erlebt er dort Vater und Mutter, die ersten "Grundgestalten" also, sowie Bruder und Schwester. Verantwortete Elternschaft setzt u.a. voraus, daß die Eltern selbst einen solchen Reifungsstand besitzen, um ihre Kinder "lebenstüchtig" machen zu können. Zu dieser "Lebenstüchtigkeit" gehört vornehmlich die Fähigkeit zur Kommunikation. Um "Kommunikation" vollziehen zu können, muß ein Mensch gelernt haben, was Unterscheiden und Entscheiden bedeutet. Diese beiden Fähigkeiten, Unterscheiden und Entscheiden, sind nach dem Heinen-Modell gleichsam die beiden "Säulen", auf denen das Gewissen aufruht? ihm jene von Mutter und Vater eingeübte Fähigkeit des Unter- und Entscheidenkönnens: das Kind lernt durch die Mutter ( $=$ maternal) die Wertsensibilität ( $=$ unterscheiden von "gut" und "böse"); das geschieht vor allem durch Liebkosungen, Erweisen von verschiedenen Formen von Zärtlichkeiten, aber auch von pädagogisch verantwortbaren Bestrafungsarten: Das Kind gewinnt ein Gespür dafür, was aus der Sicht der Mutter, mit der es ja zeitlich mehr als mit dem Vater zusammen ist, "gut" und "böse" bedeutet. Die zweite "Säule" des Gewissens ist das - keineswegs ausschließlich (!) durch die Hilfe und durch das Vorbild des Vaters eingeübte "Entscheiden" und die Fähigkeit, diese durchzuhalten. Beides, die Fähigkeit zum Unter- und Entscheiden, sind zentrale Merkmale zur Kommunikation. Ist jemand später im Leben unfähig zur Kommunikation, so hat das nach Heinen seine Ursache im ungenügenden Erleben von Vater und Mutter. Für die Pastoral ergibt sich hier die Forderung, diese Zusamenhänge zu verstehen und einem solchen Menschen personengemäße Versöhnungshilfen zu ermöglichen. Darüber hinaus sind die Grundgestalten "Vater" und "Mutter" für folgende Bereiche von Bedeutung:

Mutter:- als Ursprung, Schicksal und Helferin des Kindes;

- als Vorbild für „Häuslichkeit”;

- als Helferin, damit das Kind vom Trauen, Zutrauen, Vertrauen zum Glauben an Gott gelangen kann. in diesem Sinne wird die Mutter-Kind-Relation als "religio prima" gesehen.

Vater: - als Symbol der richtigen Autorität und Garant der Ordnung. In diesem Sinne ist der Vater auch Helfer zu Eigenständigkeit und Freiheit;

- als Repräsentant der Außenwelt und des Berufslebens, der den Jugendlichen in die „Welt” einführt, ihm bei der Berufswahl und Berufsausbildung hilft sowie ihn zur Gründung eines eigenen Hausstandes ermutigt,

- als Helfer bei der Entfaltung von Glauben und Glaubensgehorsam. 
Wenn heutzutage viele Menschen ein gestörtes Verhältnis zu den Vater- und Muttergestalten in Kirche und Staat haben, so bleibt nach Heinen die Frage, ob die derzeitige Pastoral sich genügend um die Vorbereitung gesunder Ursprungsfamilien bemüht, bestehende Familien begleitet und gefährdete Familien familiengerechte Hilfen erhalten. Wenn es stimmt, daß diese gestörten Verhältnisse zu den Vater- und Muttergestalten sich vor allem in Störungen der Kommunikation zeigen, so ist die Rückbesinnung auf die Grundgestalten „Vater" und „Mutter” unerläßlich: in der Begegnung mit ihnen wird Kommunikation eingeübt.

\section{Das brüderliche und schwesterliche Element in der Familie}

Nach dem Heinen-Modell ist das Erleben von Bruder und Schwester in zweifacher Weise von Bedeutung:

(1) Soziales Verhalten wird eingeübt: es geht um die „wie-Fragen": Wie lemt der Mensch den Umgang mit Besitz? Wie lernt der Mensch, was Teilen, Abgeben, Opfern bedeutet? Wie lernt der Mensch, mit Frustration (= Lust-Verlust) umzugehen? Wie lemt der Mensch, soziales Verhalten so einzuüben, daß man geachtet und ernstgenommen wird? Wie lernt er, was „Genießen” bedeutet? Während also im Erleben von Vater und Mutter der Mensch Hilfen bekommt, damit er später weiß, wie Glauben, Hoffen und Lieben eingeübt und entfaltet werden und was dabei etwa das „Urvertrauen" bedeutet, so sind die Geschwister von Bedeutung, wenn es darum geht, die Grundlagen der von Vater und Mutter eingeübten Kommunikation weiterzuentfalten. Konkret: wer später im Leben nie genießt, wird nicht nur, wie es der Volksmund sagt, auf die Dauer ungenießbar; er zeigt vielmehr auch an, daß er von seinen Geschwistern in diesem Punkt enttäuscht worden ist. Für die Pastoral stellen sich hier Forderungen: Wird genügend betont, daß die Eltern ihrem Kind Geschwister ,schuldig” sind? Mit der Problematik des Alleinkindes hat sich Heinen mehrfach auseinandergesezt $^{8}$. Ist sich die Pastoral bewußt, daß all die Formen der Werbung, die dem Menschen in den verschiedensten Weisen Werte, Besitz, Ansehen und Genuß zu vermitteln vorgeben, oft ausschließlich eigene kommerzielle Vorteile wittern? Erinnert sei daran, daß es eine schwere Kommunikationsstörung ist, wenn jemand mit den prinzipiell berechtigten Wünschen nach Besitz, Ansehen und Genießen nie umgehen gelemt hat. Wer aber im Sinne des Heinen-Modells eine "gesunde Ursprungsfamilie" erleben durfte, wer also nicht enttäuscht wurde von den „Grundgestalten”, dürfte gegenüber vielen Formen der Werbung immun sein.

(2) Bruder und Schwester helfen bei der „Ablösung” vom Elternhaus. Es ist ein Unterschied, ob eine Mutter oder ein Vater zum zweijährigen Sohn „mein Wilfried” oder zur Tochter „meine Monika” sagt oder zwanzig Jahre später. Während im Augenblick der Geburt die erste (= körperliche) Ablösung der Tochter bzw. des Sohnes von der Mutter erfolgt, steht die „zweite Ablösung” (Heinen vermeidet strikt das Wort „Trennung”!) noch bevor: diese „Zweite Ablösung” geschieht dann, wenn der Sohn oder die Tochter eine eigene Ursprungsfamilie gründen oder, was für die Pastoral keineswegs unbedeutend ist, sich zu einem Leben in den ehefreien Diensten des Priesterund Ordenslebens entschließen. Das setzt voraus, daß durch das personengemäße Erleben der Schwester und des Bruders die stufenweise, allmähliche Ablösung von der Ursprungsfamilie eingesehen, bejaht und gefördert wird. Das stellt viele Eltern vor schwierige Aufgaben. Die Pastoral sollte mit ihren Mitteln die Eltern bei diesem Erkenntnis- und Vollziehungsprozeß unterstützen: es geht um Kommunikation, es geht darum, daß Sohn und Tochter nicht nur untereinander und mit den Eltern „kommuni- 
zieren" lernen, sondern das Gelernte anwenden, um in einer späteren Ehe, oder in den ehefreien Diensten des Priester- und Ordensstandes und darüberhinaus in Kirche, Staat, Gesellschaft und Gemeinschaften „kommunizieren” zu können.

In diesem Zusammenhang sei darauf aufmerksam gemacht, daß das berühmte Bild der Pietà (schmerzhafte Mutter) des Michelangelo gesehen werden kann als das künstlerisch dargestellte, zeitlos gültige Bild der „Ablösung des Sohnes von der Mutter”: Maria, die Mutter hält ihren Sohn Jesus in dem Augenblick der „Ablösung” in ihren Armen. Mitbedenken sollte man hier auch die „Seligpreisung der Trauernden”(Mt 5,4): Trauer ist das Gegenteil von Schmerzverdrängung. Wer fähig zur Trauer ist, zeigt insofern Reife, weil er Schmerz auszudrücken weiß, was wiederum eine Form von Kommunikation ist; damit ist die Möglichkeit zur Schmerz-Verarbeitung gegeben.

Nach dem Heinen-Modell wird der Prozeß vom Sohn-/Tochter-Sein über das Erleben von Vater und Mutter, Bruder und Schwester hin zum Sohn-/Tochter-Haben verdeutlicht. Mit anderen Worten: das Modell zeigt auf, wie jemand, der Vater und Mutter „hat”, dazu kommt, daß er Vater bzw. Mutter „ist”.

\section{Kommunikationsstörungen}

Erhebliche Reifungsrückstände und Reifungsschwierigkeiten treten nach diesem Modell dann auf, wenn ein Mensch enttäuscht wurde oder enttäuscht wird von einigen oder allen ,acht Grundgestalten” oder von deren Stellvertretern.

Es scheint sogar so zu sein, daß jeder Mensch über eine Art „Phantom-Nerv” verfügt, der wiederum beim Ausfall einzelner, mehrerer oder aller ,acht Grundgestalten” in Form eines „Phantom-Schmerzes” einen Leidensdruck erzeugt, der das ungenügende Erleben der „acht Grundgestalten” signalisiert und anzeigt, daß ein solcher Mensch, der gestört ist in seiner Fähigkeit zur Kommunikation, sich eindringlich, unaufhörlich und auf vielfache Weise bemerkbar machen möchte, weil er sich danach sehnt, endlich mit den „Grundgestalten” oder deren Stellvertretern, von denen er enttäuscht wurde bzw. wird, ausgesöhnt zu werden.

In diesem Sinne ist das zentrale Anliegen Jesu, nämlich Versöhnung, das Ziel, das dieses Modell ereichen möchte. Mit anderen Worten: jeder Mensch stellt sich irgendwann die Frage, ob sein Leben, wenn er es so weiterlebt wie bisher, gelingen oder scheitern wird. Nie ist ein solcher Mensch zufrieden mit den Antworten, die ihn einseitig auf bessere Strukturen verweisen, ihn mit zusätzlichen Informationen füttern oder die Utopien, ,paradiesischer Systeme" verherrlichen; es geht stets um das personengemäße Erleben der „acht Grundgestalten” oder deren Stellvertreter (z.B. Großvater, Großmutter, Tante, Onkel, Vetter, Cousine etc.). Erst dann, wenn ein kommunikationsgestörter Mensch um die Ursachen seiner Störung weiß und sich um eine personengemäße Aussöhnung bemüht, wird er fähig zur Kommunikation, und der Prozeß seines lebenslangen Werde- und Reifungsprozesses kann gelingen.

\section{Direkte und indirekte Fragen}

Die Frage nach dem Wie des Gelingens der Kommunikation und somit des Lebens wird nach dem Heinen-Modell vor allem in zweifacher Weise gestellt: einmal „direkt” (z.B. vertrauensvolle Gespräche mit Seelsorgern, Eltern, Geschwistern, Ehegatten, Verwandten etc.), zum anderen aber, und heute viel häufiger, „indirekt”. Formen dieser „indirekten Frage ${ }^{9}$ sind u.a.: plötzlicher Leistungsrückgang in Schule, Beruf und 
Haushalt; Flucht in die verschiedenen Formen von Krankheit, Tabletten, Drogen, Langeweile und übertriebene Geschäftigkeit; dann die vielen Formen von Delikten, Vergehen und Verbrechen (z.B. Kaufhausdiebstahl, Raub, Erpressung, Entführung, Vergewaltigung); viele Formen der Aufsässigkeit gegenüber Elternhaus, Schule, Kirche, Staat und Gesellschaft. Konkret: ist es nicht möglich, daß ein Kind deshalb „ungehorsam" ist, weil es ,instinktiv" spürt, wie sehr es ständig vom Vater enttäuscht wird, einfach, weil der Vater unreif ist und nicht weiß, was er seinem Kind „schuldig” ist? Wäre in diesem Fall der „Ungehorsam” des Kindes nicht der Versuch, die gestörte Kommunikation zum Vater in Form gleichsam eines Verzweiflungsschreis heilen zu wollen: Vater, benimm Dich endlich so, daß Du mich nicht mehr enttäuschst! Denke daran: wenn Du mich nicht „kommunikations-fähig” machst - dann bleibe ich lebensuntüchtig und einsam ( $=$ das Gegenteil von kommunikationsfähig)!

All diese und andere Formen der „direkten” und „indirekten Frage” haben eine gemeinsame Wurzel: Grundgestalten haben enttäuscht! Was wird gefordert? Kommunikation, damit Versöhnung ermöglicht und erhalten werden kann. So werden nicht nur die familiären Gestalten wie Vater, Mutter usw., sondern auch die "Grundgestalten” maternaler und paternaler Art in Kirche, Staat, Gesellschaft aufgefordert, sich endlich so zu verhalten, daß die berechtigten Erwartungen und Forderungen ihnen gegenüber erfüllt werden, vor allem, wenn man von ihnen enttäuscht worden ist oder wird.

\section{Pastorale Überlegungen}

Hier können viele pastorale Überlegungen geschehen: Menschen, deren Fähigkeit zur Kommunikation unentfaltet oder gestört ist, weil sie enttäuscht wurden von den „acht Grundgestalten” und deshalb in den vielfachen Formen der „direkten” und "indirekten" Frage nach Hilfe suchen, fordern geradezu heraus, daß auf sie genau hingehört wird, weil vordergründige Antworten hier völlig fehl am Platz sind. Eine wichtige pastorale Hilfe liegt darin, daß solche Menschen in liebender Grundhaltung erfahren, von welchen Grundgestalten sie enttäuscht wurden und was geschehen muß, damit die personengemäße Kommunikation (= Begegnung) mitdiesen Grundgestalten ermöglicht wird.

\section{Bindung - Ablösung - Wandlung}

Nach dem Heinen-Modell sind sowohl für das Gelingen der Ehe als auch für das Gelingen der ehe-freien Dienste im Priester- und Ordensstand die personengemäße Bindung und die altersgemäße Ablösung enscheidend. Jedes Leben geschieht kommunikativ. Wenn es um das „gesamte Gelingen” dieser Kommunikation geht, so darf die Frage nach dem kommunikativen Sinn des Todes nicht ausgeklammert werden. Nach Heinen ergibt sich folgende Argumentationsfigur: jedesmal, wenn die personengemäße Bindung reift, geschieht gleichsam als Krönung die „Ablösung”, was wiederum Voraussetzung ist für neue Kommunikationen; es sei erinnert an die Tränen vieler Mütter bei der Hochzeit des Sohnes bzw. der Tochter, die spätestens dann vergessen sind, wenn diese „schmerzhafte Mutter" die Notwendigkeit ihres Schmerzes verstanden und bejaht hat und - ihren Enkel oder ihre Enkelin in den Armen hält. Nach Heinen kann das eine Zusatzhilfe sein, um zeitlebens bewußt einzuüben, was im Tod, jener „Silvesternacht des Lebens” geschieht, dann, wenn wir von allem „abgelöst” werden: wird hier nicht deutlich, was „Wandlung” meint? Wandlung ist für den katholischen Christen eine Beschreibung dessen, was bei jeder heiligen Messe geschieht. Nach dem Heinen- 
Modell darf und muß der persönliche Tod bewußt eingeübt werden. Das geschieht überall dort, wo der Mensch sich um eines „höheren Gutes” willen trennt von Personen und Sachen: Kommunikationen werden aufgegeben, um andere, qualitativ höhere erleben zu können. Kann nicht in diesem Sinne der Tod eingeübt werden als Gegenteil von Ex-Itus (= Ausgang, Ende) und somit als Trans-Itus (= Wandlung, Übergang in das unverhüllte, göttliche Leben). Durch sein Leben, Sterben und Auferstehen hat Jesus Christus denen, die glauben, ewiges Leben verheißen ${ }^{10}$. Diese Lebensgemeinschaft beginnt in den Initiationssakramenten Taufe, Firmung und Eucharistie und wird entfaltet und vollendet in den übrigen Sakramenten. Nach dem Heinen-Modell ist der Tod die höchste Form der Taufe, der Firmung und der hl. Messe (= Wandlung): alles, was an „Kommunikation” (= Begegnung und Beziehung) eingeübt wurde, war und ist angesichts des eigenen Todes nicht ohne Sinn. Was ist denn die Lebensgemeinschaft mit Gott anderes als Kommunikation? Diese höchste Form der Kommunikation, die in besonderer Weise durch die „hl. Kommunion” genährt wird, ist dann der eigene Tod: die unverhüllte Lebensgemeinschaft ( $=$ Kommunion, Begegnung, Kommunikation) mit Gott beginnt.

\section{Anmerkungen}

1 Meyers Konservations-Lexikon, Leipzig 1885 ff; Bd. IX, S. 986.

${ }^{2} \mathrm{H}$. Günther, C. und R. Willeke: Grundlegung einer bejahenden Erziehung, Affirmative Didaktik, München ${ }^{2} 1978$, S. 50.

${ }^{3}$ Inter Mirifica 4.

${ }^{4}$ Herausgeber: Sekretariat der Deutschen Bischofskonferenz, D-5300 Bonn.

5 Das Wort „Heinen-Modell” wurde von mir in mehreren Aufsätzen versandt, u.a.: Rheinischer Merkur, Köln, 27/1978, S. 11; Deutsche Tagespost, Würzburg, 27/1978, S. 11; Österreichisches Klerusblatt 16/17/1978, S. 7 ff; Der Dom, Paderborn 11/1978, S. 6; Kirche und Leben, Münster, 16/1978, S. 6; Communicatio Socialis 1977, S. 183-193.

6 Wilhelm Heinen: Werden und Reifen des Menschen in Ehe und Familie, Münster, ${ }^{2} 1978$.

${ }^{7}$ Wilhelm Heinen: Das Gewissen - sein Werden und Wirken zur Freiheit; bearbeitet und herausgegeben v. Hans Kramer, Würzburg ${ }^{2} 1971$; S. 94 ff.

8 Wilhelm Heinen: Um die Seele des Kindes, Hamm 1972, S. 25 ff.

9 Wilhelm Heinen in: J. Tenzler (Hrsg.): Urbild und Abglanz, Beiträge zu einer Synopse von Weltgestalt und Glaubenswirklichkeit, Regensburg 1972, S. 240.

10 Joh 17,24 . 


\section{SUMMARY}

Basic human values and attitudes are developed in the family. According to 'Inter Mirifica' responsible communication is impossible where conformity to basic values is lacking. The ability to communicate is learned in encounters with 'reference persons', eight basic human relationships, which Professor Heinen calls "Grundgestalten". These are father, mother, brother, sister, wife, husband, son, daughter - or their substitutes. According to the 'Heinen model', communication is distorted where any one of these relationships is lacking. Personality is damaged, and seeks to compensate either directly or indirectly, by discussions, or by expressing disillusion in the form of disobedience, diminishing energy, withdrawal through sickness or drugs. Pastoral work must interpret these reactions as the desire to attain maturity, and should aim at healing the disillusioned basic human relationships in order to make genuine human communication possible.

\section{RÉSUMÉ}

On étudie des valeurs et des attitudes fondamentales dans des familles d'origine saines. Selon "Inter Mirifica", il n'est pas de communication possible sans connaissance et accord des valeurs et des attitudes fondamentales. On étudie la facilité des communication dans la rencontre avec des personnes de liaison que le Prof. Heinen, Münster, à l'aide d'observation pendant des années, articule en " 8 formes fondamentales": père, mère, frère, soeur, époux, épouse, fils, fille ou leur représentants. Selon le "modèle des Heinen", il y a trouble des communication là où chacune des "8 formes fondamentales" déçoit. Un homme ainsi handicapé s'efforce de deux façons à reconstruire la faculté de communication: d'une part, dans la forme de "question directe" (dialogue) et dans la forme de "question indirecte" (par exemple: déobéissance, baisse de rendement, fuite dans la maladie et dans la drogue). Les efforts pastoraux doivent, en tant que sens de ces formes en question, comprendre la misère de la maturité et en fonction de cela rendre possible des aides pour la réconciliation des formes fondamentales, afin que la capacité de communication soit atteinte.

\section{RESUMEN}

Los valores y actitudes fundamentales se aprenden en el seno de la familia. Según "Inter Mirifica", ta comunicación es imposible sin conocimiento y compaginación de los valores y las actitudes fundamentales. La capacidad de comunicación se desarrolla en contacto con personas de confianza, que el profesor Heinen, Münster, clasificó, tras años de observación, en "ocho formas básicas": padre, madre, hermano, hermana, marido, mujer, hijo, hija ó sus sucedáneos. Según el "modelo Heinen" surgen problemas de comunicación social cuando decepcionan alguna de las "ocho formas básicas". Una persona lastimada de este modo, busca desarrollar capacidad de comunicación de dos maneras: en forma de "pregunta directa" (conversación) ó "preguntando indirectamente" (por ejemplo: con desobediencia, poca eficacia, huida en la enfermedad y las drogas). Los efuerzos pastorales deben considerar la falta de madurez como expresión de estas formas interrogativas y possibilitar la ayuda correspondiente para la reconciliación con las "formas básicas" decepcionantes, a fin de que se alcance la capacidad de comunicación. 